\title{
Wavelet Packet Analysis of Ground-Penetrating Radar Simulated Signal for Tunnel Cavity Fillings
}

\author{
Sheng Zhang ${ }^{1,2, *}$, Yongsuo $\mathrm{Li}^{1}$, Guihai $\mathrm{Fu}^{1}$, Wenchao $\mathrm{He}^{3}$, Da $\mathrm{Hu}^{1}$ and $\mathrm{Xin} \mathrm{Cai}^{1}$ \\ ${ }^{1}$ School of Civil Engineering, Hunan City University, Yiyang 413000, China \\ ${ }^{2}$ Smart Materials and Structures Laboratory, Department of Mechanical Engineering, University of Houston, Houston, TX 77204, USA \\ ${ }^{3}$ School of Civil Engineering, Changsha University of Science \& Technology, Changsha 410114, China
}

Received 19 October 2018; Accepted 29 December 2018

\begin{abstract}
Quality defects, such as noncompactness, cavity, and water leakage, typically appear behind tunnel linings given the lack of standardization and loose management in tunnel construction. Thus, a method for acquiring characteristic information from tunnel lining cavity fillings using frequency band energy of wavelet packet transform (FBEWPT) was proposed in this study to master the reflection characteristics of ground-penetrating radar (GPR) signal. A forward simulation of tunnel lining cavity fillings, including air, wet clay, silt, and water, was explored through finite-difference time-domain method. The spectrum characteristics of tunnel cavity fillings were analyzed from time, frequency, and time-frequency domains. Results demonstrate that amplitude reflection clearly occurs at the inhomogeneous interface when GPR electromagnetic waves collide with the tunnel lining cavity fillings. However, the degree of attenuations is different considering the various dielectric constants of objects. These relative dielectric constants reveal the reflection interface position and other information between lining and fillings. With the increase in the difference in relative dielectric constants for lining cavity fillings, the attenuation of FBEWPT for GPR signals increasingly strengthens, and the variance and standard deviation of the FBEWPT for GPR signals constantly decrease, thereby reflecting the strong reflection and attenuation of GPR signals that appear in the propagation process. This study provides a favourable reference for obtaining GPR signal spectrum interpretation for tunnel lining cavity fillings in actual projects.
\end{abstract}

Keywords: Tunnel engineering, Cavity fillings, Ground-penetrating radar, Wavelet packet analysis, Finite-difference time-domain method

\section{Introduction}

With the further development of "One Belt, One Way" strategy and Western Development Strategy in China and the further input of the national infrastructure construction, Chinese highway and railway transportation networks have developed at an unprecedented speed. A transportation network typically consists of many engineering buildings, including roads, bridges, and tunnels. A tunnel is an important and controllable section in the network. However, many tunnels are built in complex topography, geomorphology, and geological environment to avoid largescale excavation of mountains and protect ecological environment. Approximately $10,000 \mathrm{~km}$ tunnels have been built in the railway field from 2008 to 2020 . China has the largest and the most tunnel projects in the world, with the most complex geological conditions and structural forms, and the fastest development of construction technology [1]. In tunnel construction, quality problems, such as noncompactness, cavity, and water leakage, appear behind the tunnel lining given the complex geological conditions, harsh construction environment, lack of standardization, and loose management [2]. The quality problems of tunnel lining are key factors that hinder the rapid development of tunnel projects. Lining cavity, which is a major obstacle of tunnel

*E-mail address: zhangsheng0403311@163.com ISSN: $1791-2377$ @ 2018 Eastern Macedonia and Thrace Institute of Technology. All rights reserved. doi:10.25103/jestr.116.09 lining, is considered an important content in completing tunnel acceptance and checkout testing. Thus, the quality inspection of lining structures must be strengthened to ensure the safety and durability of tunnel lining and the overall stability and reliability of tunnel structures.

Ground-penetrating radar (GPR) detection method is extensively used in the field of tunnel lining quality detection given its flexible operation, high efficiency, nondestructiveness, and high resolution [3-5]. The quality problems of tunnel lining, such as noncompactness and cavity, are determined through the GPR method. We posit that a huge difference in relative dielectric constant exists between lining defects and lining concrete, and the electromagnetic wave emitted by GPR main engine is reflected when it encounters lining quality defects during the propagation of lining concrete. Moreover, the reflected signal is received and recorded using a receiving antenna. However, a poor imaging quality of GPR images is produced in practical detection because on-site acquisitions of GPR signals are affected by factors, such as site limitation, detection conditions, and various noises. The existence and location of quality defects can only be detected in tunnel lining. Accurate information on the properties of tunnel lining quality defects is difficult to provide. Forward simulation and analysis of the GPR detection signals of tunnel lining cavity fillings are necessary to determine the attributes of cavity fillings behind tunnel lining. The proposed method provides a scientific basis for on-site GPR detection work. 
Finite-difference time-domain (FDTD) method is a wave equation method based on Maxwell equation. In comparison with other numerical methods, the FDTD method can calculate the time domain directly, save more computing time, and leave more storage space. This method has achieved favorable results and has been extensively used in the forward simulation of GPR [6-7]. The forward simulation of the cavity fillings of tunnel lining is analyzed through the FDTD method. The signal response characteristics of GPR signals for cavity fillings of tunnel lining are studied. The frequency band energy of wavelet packet transform (FBEWPT) method is used to analyze single-GPR analog signals in time, frequency, and timefrequency domains. The identification degree of GPR detection signals of different cavity fillings of tunnel lining is improved. This study provides a geological interpretation basis for in situ image identification of the cavity fillings of tunnel lining.

\section{State of the art}

Previous studies of quality defects detection for tunnel lining have mainly focused on three aspects. The first aspect is the signal-processing method of the GPR detection data in the field. The reflection model of a GPR electromagnetic wave for tunnel lining detection is applied, and the expression of a reflection coefficient spectrum in generalized domain is deduced by analyzing the propagation law of an electromagnetic wave in tunnel lining. A method is proposed on the basis of a reflection coefficient spectrum to estimate the thickness and height of cavity for tunnel lining. A study has provided the basis for automatic recognition technology of radar images for subsequent tunnel lining detection [8]. On the basis of the multidimensional analysis of GPR reflection signal, Gao et al. proposed a new approach to intelligent identification of tunnel defects. A two-class model of defect signals was constructed by training typical features using a support vector machine algorithm. The proposed approach realized the automatic identification of the horizontal distribution range of defect and provided feasible ideas for designing intelligent identification algorithms for different types of detection targets [9]. Many studies have mainly focused on the reflection characteristics of electromagnetic waves in thin layers. The reflection coefficient of thin layers was deduced by Zhang et al. by establishing the layered medium model with thin layers; the Hilbert spectral analysis method was introduced to identify and analyze the measured GPR data. This model is favorable for improving the capability of GPR horizon analysis [10]. To satisfy the requirements of tunnel defect survey and regular inspection, Zan et al. developed vehicle GPR, a new method for remote detection; these authors provided a fast and noninterference method for regular inspection and health assessment of existing tunnels and ensured the safe operation of health condition detection of railway tunnels during service period [11]. On the basis of the analysis of the reflected wave signals of numerous defects in tunnels, Baryshnikov et al. proposed a complete set of optimization rules for detection parameters to study the difference in detection accuracy under various detection parameters [12]. The reflected wave image of the target body was extracted from considerable data measured by GPR to establish the sample library. The automatic identification of the detected target in the radar image was realized by matching the sample library with the fuzzy logic algorithm [13].
According to the investigation of Nanchang Metro Line 1, GPR was combined with image processing by $\mathrm{Yu}$ et al. to determine the effectiveness of grouting evaluation method. The simulation of the FDTD method was used to assist the map interpretation of GPR, which ensured the construction quality of the metro tunnel and reduced the potential operational risk [14]. Fourier time-frequency analysis [1516], wavelet analysis [17-18], and Hilbert-Huang transform [19] have been proposed to analyze GPR detection data, and many feasible ideas have also been introduced.

The second aspect is to conduct a physical simulation test for the defects that easily occur in tunnel lining structures. A large-scale model of railway tunnel lining structures was established, and physical model tests of GPR were performed by Yang et al. to address the complex defects of railway tunnel composite lining. The approach offers reference values for the practical engineering GPR detection and analysis of the results [20]. Cavities are observed behind tunnel lining because of high groundwater pressure. A method for detecting cavities in tunnel lining grouting by GPR was proposed by Kravitz et al. The image characteristics of GPR detection signals in the tunnel lining grouting area were revealed by simulating air and water embedded in the grouting area behind the tunnel lining. The field results were compared with the numerical model to validate the effectiveness of cavity detection behind tunnel lining [21]. Pre-embedding plastic balls (e.g., cavities) were buried by Lai et al. underground to find and locate defects. A blind test of nondestructive detection was applied by using GPR to study the capabilities of detection institutions that were assumed previously. The purpose of which was to help the Highways Department of the Hong Kong Government investigate hidden quality problems of traffic engineering structures. A simple Ricker wavelet model was used by Nobes et al. as an example to explain several basic attributes of the characteristic response of GPR when it encountered air, water, and other materials. For example, the top reflection was completely separated from the bottom reflection when the cavity time delay exceeded twice the wavelet pulse width. By contrast, the top and the bottom reflections of the cavity interfered with each other; thus, using GPR to infer cavity thicknesses was difficult [23].

The third aspect is to perform forward simulation in accordance with the common quality problems in tunnel lining construction. Wavelet Galerkin method was applied by Feng et al. to discretize Maxwell's two curl equations and deduced the 3D difference formula and numerical stability conditions of GPR. On the basis of DB2-multiresoultion time-domain (MRTD) algorithm, a forward simulation program of the GPR MRTD method was developed, and the reliability and accuracy of GPR detection were improved [24]. Irregular and complicated tunnel lining defects cannot be finely depicted with the standard finite difference simulation method. A new hybrid algorithm has been proposed for all-refined GPR simulation by combining the finite-element time-domain and FDTD methods. Several complicated numerical models involving different typical defects and mixed case (e.g., noncompactness, cavity, and leakage with fractures and cracks) have also been established and have provided precise technical guidance for defect interpretation [25]. For typical lining defects, the FDTD method was used by Li et al. to simulate the GPR detection of a tunnel lining defect model; these authors identified the defects quantitatively through spectrum analysis. Thus, the reliability of the forward simulation and interpretation criteria of the GPR detection of tunnel lining 
defects was verified [26]. In terms of the common point-like poor geological bodies in borehole radar exploration, such as cavities, karst caves and buried objects, etc., the forward simulation of response of the tool to point unfavorable geobodies of different surrounding rock and filling condition was investigated with the FDTD method. The influence of surrounding rock and filling condition on response characteristics of borehole radar was analyzed [27]. GPR responses expected over numerous synthetic models that resemble certain commonly encountered geotechnical structures, including a single horizontal cylinder with different fluid contents, double horizontal cylinders, and 2D arbitrary polygonal bodies, have been simulated using an improved FDTD forward modeling algorithm. The geometrical (shape, radius, and depth) and physical (target media material, filled fluids, and type of host medium) parameters are mainly studied. Mathematical relationships between the physical and geometrical parameters of models and the height-to-width ratio of hyperbola response have been determined. The corresponding geometrical parameters of such circular structures can be estimated through the characteristics of the measured hyperbola responses [28]. The abovementioned studies have mainly detected and analyzed tunnel lining quality from the field, indoor model test, and forward simulation. For example, common quality problems in tunnel lining structure have been simulated simply, but the simulated characteristic signals of GPR have not been analyzed completely when the tunnel lining structure has been simulated by FDTD method. Thus, the systematic interpretation of GPR atlas characteristics is crucial. In the present study, the FDTD method is applied to simulate the GPR forward simulation of tunnel lining cavity and fillings. The time-frequency analysis of GPR response characteristic signals for tunnel lining cavity fillings is conducted through the FBEWPT method to discuss the influence law of cavity fillings. The improvement of the identification degree of GPR detection signals of different fillings in tunnel lining cavity and the introduction of a reference guide for image feature interpretation of fillings behind tunnel linings are important phases.

The remainder of this study is organized as follows. Section 3 summarizes the FBEWPT analysis method and the GPR FDTD method for tunnel lining cavity fillings and constructs the GPR forward model of tunnel lining cavity fillings. Section 4 analyzes time-frequency characteristics of typical single-channel GPR signals through the FBEWPT method and studies the influences of energy characteristics for lining cavity fillings from time, frequency, and timefrequency domains. Section 5 summarizes the conclusions drawn from this study.

\section{Methodology}

\subsection{Method for GPR signal analysis}

\subsubsection{Time-domain analysis method}

The GPR reflected signal can be expressed as follows:

$$
x(t)=A(t) \cos \left[2 \pi f_{0} t+\theta(t)\right]
$$

where $A(t)$ is the amplitude function of the GPR reflected signal, $t$ is the recording time, $f_{0}$ is the central frequency, and $\theta(t)$ is the phase function.

\subsubsection{Frequency-domain analysis method}

In comparison with the time-domain analysis, the frequency-domain analysis of GPR reflection signal can further determine the frequency composition and distribution range. For discrete aperiodic GPR reflection signal, the corresponding discrete-time Fourier transform of Eq. (1) can be represented as follows:

$$
X_{k}=\sum_{i=1}^{N-1} x_{i} e^{-j 2 \pi i k / N}(k=0,1, \cdots, N-1),
$$

where $X_{k}$ is the discrete time spectrum of the GPR signal. The amplitude-frequency and phase-frequency relationships can be expressed as follows:

$\left|X_{k}\right|=\sqrt{\operatorname{Re}\left(X_{k}\right)^{2}+\operatorname{Im}\left(X_{k}\right)^{2}}$,

$\varphi_{k}=\arctan \frac{\operatorname{Im}\left(X_{k}\right)}{\operatorname{Re}\left(X_{k}\right)}$.

The frequency of the GPR reflected signal can be judged in accordance with the time profile waveform of the GPR. A narrow waveform indicates a high frequency; a wide waveform denotes a low frequency. However, this evaluation is only a qualitative judgment of the relative frequency of the GPR reflected signal, and the specific frequency is unknown.

\subsubsection{FBEWPT analysis method}

Wavelet transform is a time-frequency domain localization analysis method. The window size is fixed, but the shapes can be changed. The low-frequency part has high-frequency and low-time resolutions, whereas the high-frequency part has low-frequency and high-time resolutions. The GPR signal can decompose effectively in time-frequency, but the scale function used in wavelet transform is binary decomposition. Therefore, the frequency resolution of the wavelet transform is poor when the high-frequency components of the GPR signal are analyzed. In comparison with the wavelet transform, the wavelet packet analysis can further refine the high-frequency components of the GPR signal. The adaptive frequency decomposition can be performed in accordance with the inherent characteristics of the analyzed signal, thereby improving the time-frequency resolution considerably. Thus, the wavelet packet analysis has a broad application prospect.

Energy distributions for different frequency bands of GPR signals are necessary for the analysis. The characteristics of energy distributions for GPR signals represent the relationship between GPR signal energy and frequency. The original signal for GPR is decomposed by wavelet packet transform into wavelet packets. According to sampling theorem, the sampling frequency of the original signal for GPR is $F_{s}$, and its Nyquist frequency is $F_{s} / 2$. The original signal for GPR is generally decomposed into the 10th layer, and the corresponding lowest frequency band is $0-F_{s} / 2048$. If the reconstructed signal of each frequency band for GPR is $S_{10, j}$, and the corresponding energy is $E_{10, j}$, then 
$E_{10, j}=\int\left|S_{10, j}(t)\right|^{2} d t=\sum_{k=1}^{m}\left|x_{j, k}\right|^{2}$,

where $x_{j, k}\left(j=0,1,2, \cdots, 2^{10}-1, k=1,2, \cdots, m\right)$ (m refers to the discrete sampling points of GPR signals) is the amplitude of the discrete point of the reconstructed signal $S_{10, j}$.

The total energy of the original signal for GPR is set as $E_{0}$, and

$E_{0}=\sum_{j=0}^{2^{10}-1} E_{10, j}$.

The percentage of the energy of each frequency band signal and the total energy of the original signal for GPR is

$E_{j}=E_{10, j} / E_{0} \times 100 \%, j=0,1,2, \cdots, 2^{10}-1$.

Thus, from Eq. (5) to Eq. (7), the energy of different frequency bands of GPR signals can be obtained after wavelet packet decomposition. The energy change rule of GPR can accordingly be determined in the process of medium propagation.

\subsection{FDTD method for the GPR of tunnel cavity fillings}

\subsubsection{FDTD method}

In nonsource field, the two curls of Maxwell equation can be expressed as

$$
\left\{\begin{array}{l}
\nabla \times H=\varepsilon \frac{\partial E}{\partial t}+\sigma E \\
\nabla \times E=-\mu \frac{\partial H}{\partial t}-\sigma_{m} H
\end{array},\right.
$$

where $H(\mathrm{~A} / \mathrm{m})$ is the magnetic field intensity vector, $E(\mathrm{~V} / \mathrm{m})$ is the electric field strength vector, $\varepsilon$ is the dielectric constant, $\sigma(\mathrm{S} / \mathrm{m})$ is the electrical conductivity, $t$ (s) is the time, $\mu(\mathrm{H} / \mathrm{m})$ is the relative permeability, and $\sigma_{m}(\mathrm{w} / \mathrm{m})$ is the equivalent magnetic permeability.

Two curls of Maxwell equation are converted from differential or derivative into a difference in the form with the accuracy of second-order central difference in the FDTD method. The electric and magnetic fields are sampled alternately in time sequence, and the difference is half the time step. Therefore, the FDTD equations of 2D electromagnetic wave can be expressed as

$$
\begin{gathered}
E_{x}^{n+1}(i, j)=C A \times E_{x}^{n}(i, j)+ \\
C B \times \frac{H_{y}^{n+1 / 2}(i+1 / 2, j)-H_{y}^{n+1 / 2}(i-1 / 2, j)}{\Delta x}- \\
C B \times \frac{H_{x}^{n+1 / 2}(i, j+1 / 2)-H_{x}^{n+1 / 2}(i, j-1 / 2)}{\Delta y} \\
H_{x}^{n+1 / 2}(i, j+1 / 2)=C P \times H_{x}^{n-1 / 2}(i, j+1 / 2)- \\
C Q \times \frac{E_{z}^{n}(i, j+1)-E_{z}^{n}(i, j)}{\Delta y} \\
H_{y}^{n+1 / 2}(i+1 / 2, j)=C P \times H_{x}^{n-1 / 2}(i+1 / 2, j)+ \\
C Q \times \frac{E_{z}^{n}(i+1, j)-E_{z}^{n}(i, j)}{\Delta x}
\end{gathered}
$$

In Eqs. (9) and (10),

$$
\begin{aligned}
& C A=\frac{2 \varepsilon(i, j)-\sigma(i, j) \times \Delta t}{2 \varepsilon(i, j)+\sigma(i, j) \times \Delta t} \\
& C B=\frac{2 \Delta t}{2 \varepsilon(i, j)+\sigma(i, j) \times \Delta t} \\
& C P=\frac{2 \mu(i, j)-\sigma_{m}(i, j) \times \Delta t}{2 \mu(i, j)+\sigma_{m}(i, j) \times \Delta t}, \\
& C Q=\frac{2 \Delta t}{2 \mu(i, j)+\sigma_{m}(i, j) \times \Delta t}
\end{aligned}
$$

where $E_{x}$ is the electric intensity oriented in the $x$ direction; $H_{x}$ and $H_{y}$ are the magnetic intensities oriented in the $x$ and $y$ directions, respectively; $\Delta x$ and $\Delta y$ are the space steps oriented in the $x$ and $y$ directions, correspondingly; $\Delta t$ is the time step; and $(i, j)$ is the node coordinate.

The time step $\Delta t$, space step $\Delta x$, and $\Delta y$ are required to guarantee the convergence of the solution of discrete finite difference equations in a time domain. The following relations are satisfied:

$$
\Delta t \leq \frac{1}{c \sqrt{\left(\frac{1}{\Delta t}\right)^{2}+\left(\frac{1}{\Delta y}\right)^{2}}},
$$

where $c$ is the speed of light in the free space.

\subsubsection{FDTD modeling of tunnel cavity fillings}

The tunnel lining is assumed to be a continuous homogeneous material in a semi-infinite space, and the reflection and refraction of the electromagnetic wave are performed on a $2 \mathrm{D}$ plane. A geoelectric model of the cavity fillings of tunnel lining is designed to study the reflection characteristics of GPR simulated signals filled with different materials in tunnel lining cavity, as illustrated in Fig. 1.

The parameters of the model are presented as follows: (1) the range of the simulated area is $1 \mathrm{~m} \times 1 \mathrm{~m}$, the lower left angle is the origin of coordinates, the horizontal coordinates are the horizontal distance, and the longitudinal coordinates are the depth; (2) the target object is a square cavity, the size is $0.2 \mathrm{~m} \times 0.2 \mathrm{~m}$, the distance between the left side of the cavity and the left edge of the simulated area is $0.4 \mathrm{~m}$, and the buried depth is $0.1 \mathrm{~m}$; (3) the concrete dielectric constant is 6 , the conductivity is $1 \mathrm{~S} / \mathrm{m}$, and the permeability is 1 ; and (4) the fillings are air, wet clay, silt, and water. Table 1 lists the geometric and physical parameters of different fillings in tunnel lining cavity.

Table 1. Geometric and physical parameters of common materials

\begin{tabular}{l|l|l|l|l}
\hline Material & $\begin{array}{l}\text { Relative } \\
\text { dielectric } \\
\text { permittivity }\end{array}$ & $\begin{array}{l}\text { Conductivity } \\
\mathbf{( S / m )}\end{array}$ & $\begin{array}{l}\text { Wave } \\
\text { velocity } \\
\text { (m/ns) }\end{array}$ & $\begin{array}{l}\text { Attenuation } \\
(\mathbf{d B} / \mathbf{m})\end{array}$ \\
\hline Air & 1 & 0 & 0.3000 & 0 \\
Concrete & 6 & 0.01 & 0.1225 & 0.01 \\
Wet clay & 12 & $0.5-2.0$ & 0.0866 & $0.4-1.0$ \\
Silt & 49 & 0.5 & 0.0429 & 0.1 \\
Freshwater & 81 & 0.5 & 0.0333 & 0.1 \\
\hline
\end{tabular}




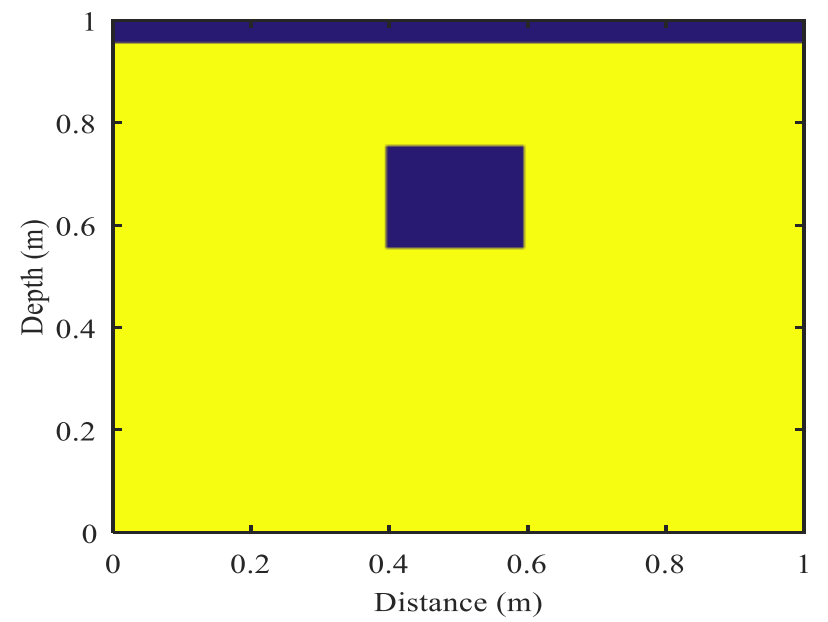

Fig. 1. Geometric model of the cavity fillings of tunnel lining

\subsubsection{Acquisition of GPR simulated signals}

The parameters used in actually detecting tunnel lining are provided as follows: the central frequency is $500 \mathrm{MHz}$, the boundary absorption condition is a perfectly matched layer, the excitation source is Ricker wavelet, the space grid step is
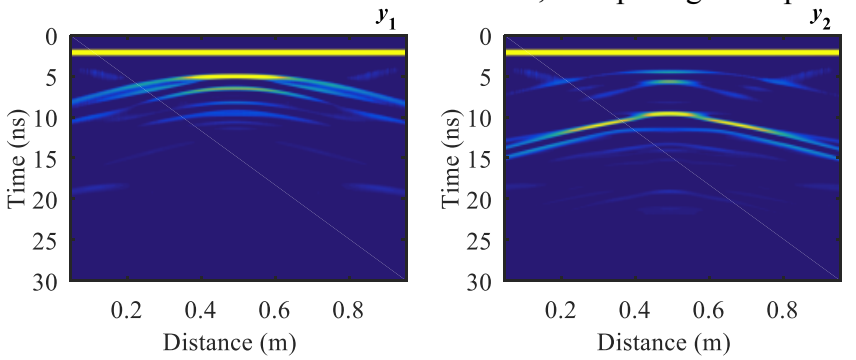

$0.0025 \mathrm{~m}$, the sampling step is $0.0025 \mathrm{~m}$, the sampling path is 365 , and the total sampling time is $30 \mathrm{~ns}$. Each working condition is marked in the acquisition process. For example, " $y_{1}$ " represents the cavity-filling material, " $y_{2}$ " corresponds to the wet clay, " $y_{3}$ " corresponds to the silt, and " $y_{4}$ " corresponds to the water. The GPR forward modeling of tunnel lining cavity filled with different materials is performed through the FDTD method in the present study. The time profile of GPR is depicted in Fig. 2. In Fig. 2, (1) when GPR electromagnetic wave encounters different materials, such as cavity, wet clay, silt, and water, a strong amplitude reflection occurs at the heterogeneous interface; (2) the propagation time of electromagnetic wave signals in different materials is different. With the increase in the dielectric constant of the lining cavity fillings, the propagation time of the GPR electromagnetic wave in the lining cavity fillings will be prolonged, and the amplitude attenuation of the GPR signal will also be increased. The properties of the tunnel lining cavity fillings, however, cannot be judged. Therefore, other methods must be adopted for further analysis.
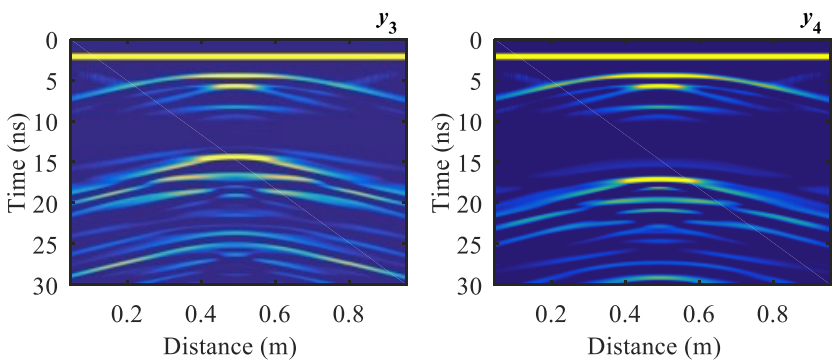

Fig. 2. Time sections of the GPR-simulated signals with different tunnel cavity fillings

\section{Result analysis and discussion}

During testing of the quality of tunnel lining, we find cavities behind the lining concrete, and negative geological phenomena, such as wet clay and silt fillings, occur in the cavities. For these geological phenomena, the GPR detection signals have distinct response characteristics, which are mainly manifested in the waveform, frequency, and reflection energy differences of GPR atlas.
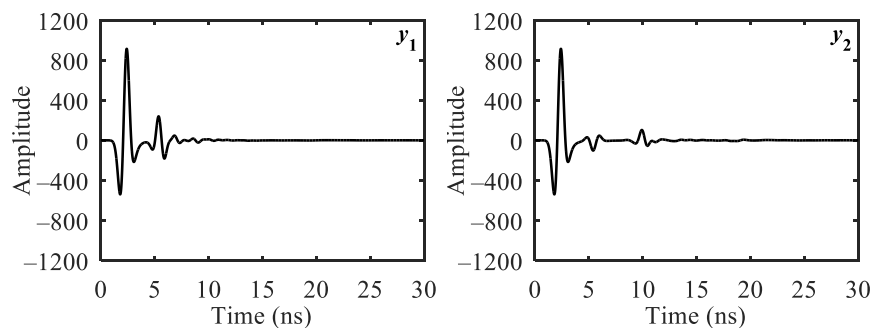

\subsection{Results of time-domain analysis}

The typical waveforms under each working condition are compared and analyzed in a time domain to illustrate the image characteristics of the GPR time profiles when electromagnetic waves encounter different materials. The middle signal is extracted from the GPR time profile demonstrated in Fig. 2. The typical single-channel GPR signal is exhibited in Fig. 3.

Fig. 3. Single-channel GPR signals of the GPR-simulated signals with different tunnel cavity fillings

Fig. 3 illustrates that the reflections of different fillings in tunnel lining cavity on the single-channel GPR signal are different given apparent differences in electromagnetic properties between tunnel lining cavity fillings and lining concrete. Therefore, vibration variation in a single-channel GPR signal can reflect important information, such as the reflection position of an electromagnetic wave on the interface between lining concrete and cavity fillings in the time domain.
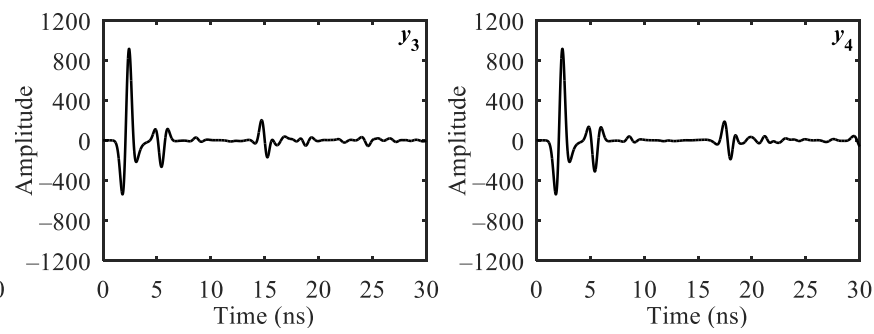

\subsection{Results of frequency-domain analysis}

The quality of tunnel lining is tested by the GPR. The electromagnetic wave of the GPR is reflected when it collides with different interfaces with various dielectric constants. The relevant information of detecting target bodies was taken along by the reflected electromagnetic wave signal. Differences in the frequency and energy attenuation of the electromagnetic reflection signal occur given the different dielectric properties of the target. 
Sheng Zhang, Yongsuo Li, Guihai Fu, Wenchao He, Da Hu and Xin Cai/

Journal of Engineering Science and Technology Review 11 (6) (2018) 62 - 69

Therefore, the spectrum of GPR shows different reflection

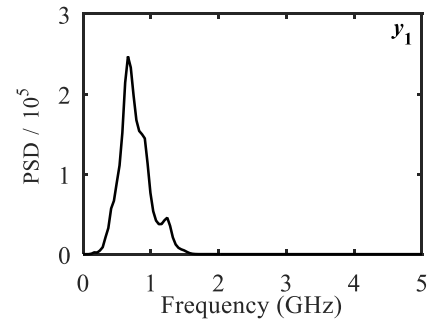

characteristics after the spectrum analysis of the GPR signal.

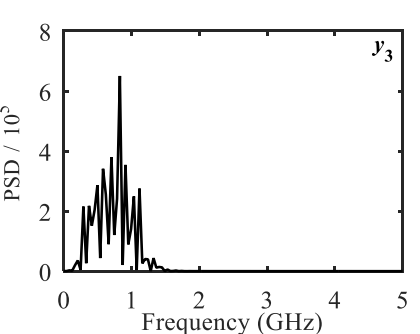

Fig. 4. Frequency spectrums of a single-channel GPR signal

The spectrum analysis of the single-channel GPR signal displayed in Fig. 3 is performed in the frequency domain. The results are presented in Fig. 4. The frequency components, such as cavity, wet clay, silt, and water, of the GPR signal are less than $2 \mathrm{GHz}$ when the electromagnetic wave of the GPR collides with the medium, and the frequency components are relatively concentrated. Considerable oscillations of the GPR signal in the frequency occur with the increase in the dielectric constant of lining cavity fillings. That is, an increase in the attenuation of the GPR signal indicates that multiple reflection waves exist at the upper and lower interfaces of tunnel lining cavity fillings.

\subsection{Results of FBEWPT analysis}

The same GPR signal handled different wavelet bases generates various results because the wavelet basis in the wavelet analysis is neither arbitrary nor unique. Therefore, selecting the optimal wavelet basis function is an important step in analyzing and processing the GPR detection signal by wavelet packet transform. Numerous experiments and study results show that Daubechies series of wavelet basis functions exhibits favorable compactness, smoothness, and approximate symmetry among many discrete wavelets. After comparing the effect of extracting band energy feature based on Daubechies series wavelet packet analysis, "db4" wavelet is selected as the wavelet basis function to extract the band energy feature of the wavelet packet transform in this study. The single-channel GPR signal is further compared and analyzed in the time-frequency domain by using the $\mathrm{db} 4$ wavelet packet analysis method. The decomposition coefficients of 1024 wavelet packets are obtained from analyzing single-channel GPR signals by "db4" wavelet at level 10th.

According to Eqs. (5)-(7), a calculation and analysis program is compiled to calculate the energy of 1024 wavelet packet decomposition coefficients. The percentage of the energy of reconstructed signals in each frequency band is listed in Table 2. The energy distribution of reconstructed signals in each frequency band is plotted in Fig. 5.

Table. 2. Energy and percentage of different frequency bands for single-channel GPR signal

\begin{tabular}{|c|c|c|c|c|c|c|c|c|c|c|c|}
\hline \multirow{2}{*}{ No. } & \multirow{2}{*}{ Frequency bands $(\mathrm{GHz})$} & \multicolumn{4}{|c|}{ Analyzed signal } & \multirow{2}{*}{ No. } & \multirow{2}{*}{ Frequency bands $(\mathrm{GHz})$} & \multicolumn{4}{|c|}{ Analyzed signal } \\
\hline & & $y_{1}$ & $y_{2}$ & $y_{3}$ & $y_{4}$ & & & $y_{1}$ & $y_{2}$ & $y_{3}$ & $y_{4}$ \\
\hline 1 & $0-0.08$ & 0.01 & 0.00 & 0.07 & 3.25 & 21 & $1.66-1.74$ & 0.41 & 0.11 & 0.19 & 0.19 \\
\hline 2 & $0.08-0.17$ & 0.16 & 0.25 & 0.36 & 2.92 & 22 & $1.74-1.82$ & 0.18 & 0.30 & 0.16 & 0.28 \\
\hline 3 & $0.17-0.25$ & 1.34 & 3.32 & 6.32 & 2.84 & 23 & $1.82-1.90$ & 0.01 & 0.01 & 0.02 & 0.01 \\
\hline 4 & $0.25-0.33$ & 0.70 & 0.89 & 1.41 & 3.25 & 24 & $1.90-1.99$ & 0.01 & 0.06 & 0.02 & 0.01 \\
\hline 5 & $0.33-0.41$ & 21.10 & 16.23 & 21.24 & 14.87 & 25 & $1.99-2.07$ & 0.23 & 0.07 & 0.35 & 0.23 \\
\hline 6 & $0.41-0.50$ & 2.39 & 16.41 & 12.88 & 12.18 & 26 & $2.07-2.15$ & 0.24 & 0.34 & 0.36 & 1.25 \\
\hline 7 & $0.50-0.58$ & 0.75 & 3.99 & 8.14 & 3.76 & 27 & $2.15-2.24$ & 0.93 & 1.15 & 0.97 & 1.34 \\
\hline 8 & $0.58-0.66$ & 7.73 & 11.49 & 9.35 & 9.42 & 28 & $2.24-2.32$ & 0.27 & 0.57 & 0.74 & 0.58 \\
\hline 9 & $0.66-0.75$ & 2.57 & 2.48 & 1.76 & 1.12 & 29 & $2.32-2.40$ & 0.13 & 0.07 & 0.08 & 0.13 \\
\hline 10 & $0.75-0.83$ & 7.73 & 4.61 & 3.26 & 2.67 & 30 & $2.40-2.48$ & 0.45 & 0.16 & 0.18 & 0.38 \\
\hline 11 & $0.83-0.91$ & 12.55 & 14.71 & 7.24 & 12.57 & 31 & $2.48-2.57$ & 0.31 & 0.45 & 0.54 & 0.24 \\
\hline 12 & $0.91-0.99$ & 0.77 & 2.94 & 2.55 & 3.07 & 32 & $2.57-2.65$ & 0.27 & 0.21 & 0.65 & 0.20 \\
\hline 13 & $0.99-1.08$ & 21.42 & 3.04 & 3.60 & 8.38 & 33 & $2.65-2.73$ & 0.00 & 0.00 & 0.00 & 0.00 \\
\hline 14 & $1.08-1.16$ & 13.64 & 10.55 & 4.54 & 12.10 & 34 & $2.73-2.82$ & 0.00 & 0.00 & 0.00 & 0.00 \\
\hline 15 & $1.16-1.24$ & 1.97 & 1.04 & 5.98 & 0.90 & 35 & $2.82-2.90$ & 0.00 & 0.00 & 0.00 & 0.00 \\
\hline 16 & $1.24-1.32$ & 1.55 & 4.40 & 6.94 & 1.68 & 36 & $2.90-2.98$ & 0.00 & 0.00 & 0.00 & 0.00 \\
\hline 17 & $1.32-1.41$ & 0.00 & 0.00 & 0.00 & 0.01 & 37 & $2.98-3.06$ & 0.00 & 0.00 & 0.00 & 0.00 \\
\hline 18 & $1.41-1.49$ & 0.00 & 0.00 & 0.00 & 0.02 & 38 & $3.06-3.15$ & 0.00 & 0.00 & 0.00 & 0.00 \\
\hline 19 & $1.49-1.57$ & 0.02 & 0.02 & 0.01 & 0.02 & 39 & $3.15-3.23$ & 0.00 & 0.00 & 0.00 & 0.00 \\
\hline 20 & $1.57-1.66$ & 0.00 & 0.00 & 0.00 & 0.01 & 40 & $3.23-F_{\mathrm{s}} / 2$ & 0.11 & 0.10 & 0.10 & 0.09 \\
\hline
\end{tabular}
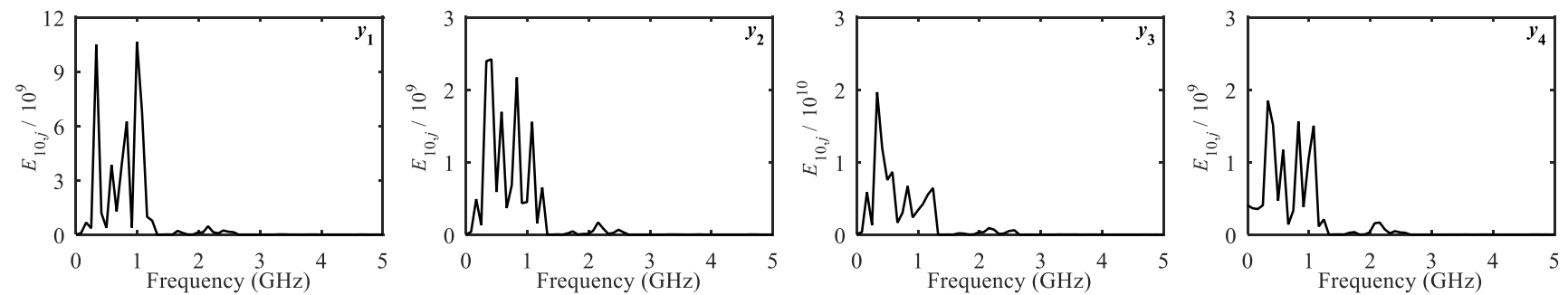

Fig. 5. Energy distribution of each frequency band for the single-channel GPR signal 
In Fig. 5 and Table 2, when the tunnel lining cavity is filled with air, wet clay, silt, and water, the GPR signals are analyzed by the FBEWPT method, and the rules are presented as follows:

(1) The total energies of different frequency bands of wavelet packet decomposition coefficients for the singlechannel GPR signal are $4.9692 \times 10^{10}, 1.4743 \times 10^{10}$, $9.2546 \times 10^{11}$, and $1.2423 \times 10^{11}$. A high difference in the relative dielectric constant between tunnel lining and cavity fillings indicates a higher attenuation of frequency band energy by wavelet packet transform for the GPR signal and a faster attenuation of the high-frequency component decays than that of the low-frequency component.

(2) The energy of the GPR signal is extensively distributed, but the energy mainly distributes in the range of 0-2 GHz. In Table 2, the energy percentages of the GPR signal in the range of $0-1.32 \mathrm{GHz}$ are $96.38 \%, 96.35 \%$, $95.64 \%$, and $94.98 \%$.

(3) Variance and standard deviation are the most common and important indicators for measuring the variation degree of discrete data in probability and statistics theory. The variance and standard deviation of energy percentages in the range of $0-1.32 \mathrm{GHz}$ are analyzed. This study shows that when tunnel lining cavity is filled with air, wet clay, silt, and water, the variances in the energy of the wavelet packet decomposition coefficients for GPR signals are 53.91, 33.72, 28.75, and 22.82; the standard deviations are $7.11,5.62,5.19$, and 4.62 . The results show that the variance and standard deviation in the energy of the wavelet packet decomposition coefficients for GPR signals decrease with the increase in the difference in relative dielectric constant between tunnel lining and cavity fillings.

\section{Conclusions}

A novel method based on the FDTD method was used to conduct forward numerical simulation of cavity fillings of tunnel lining and analyze the response characteristics of lining cavity fillings to determine the atlas relationship between the cavity fillings of tunnel lining and the frequency band energy characteristics of GPR detection signals. The following conclusions were drawn from this study:

(1) The reflection on the single-channel waveform of the GPR is different when the tunnel lining cavity is filled with various media. In the time domain, vibration changes in a single-channel waveform of the GPR can reflect the reflection position at the interface between the lining concrete and the cavity fillings.

(2) When the tunnel lining cavity is filled with air, wet clay, silt, and water, a remarkable difference in a relative dielectric constant between the lining concrete and the cavity fillings leads to a high attenuation of the FBEWPT for the GPR signal increases and fast attenuation of the highfrequency component decays.

(3) With the increase in the difference in relative dielectric constant between tunnel lining and cavity fillings, the variance and standard deviation in the energy of the wavelet packet decomposition coefficients for GPR signals decrease, thereby indicating that considerable reflection and attenuation of GPR signal occur in the propagation process of tunnel lining cavity fillings.

A new method for identifying the characteristics of the cavity fillings of tunnel lining by combining theoretical research with forward simulation was proposed in this study. The characteristics reflected in the method could be used as a reference for radar image interpretation of tunnel lining cavity fillings. The proposed method was applied to analyze and handle a model test and field test data in further study considering the lack of actual data of indoor model and field tests. Thus, interpreting radar imaging law of typical quality defects behind tunnel lining is accurate.

\section{Acknowledgements}

This work was supported by the National Natural Science Foundation of China (Nos. 51608183 and 51678226) and the Natural Science Foundation of Hunan Province in China (Nos. 2018JJ3022 and 2016JJ4013).

This is an Open Access article distributed under the terms of the Creative Commons Attribution Licence

\section{References}

1. Li S. C., Liu B., Sun H. F., Nie L. C., Zhong S. H., Su M. X., Li $\mathrm{X}$., $\mathrm{Xu} \mathrm{Z}$. H., "State of art and trends of advanced geological prediction in tunnel construction". Chinese Journal of Rock Mechanics and Engineering, 33(6), 2014, pp.1090-1113.

2. Lai W. W. L., Dérobert X., Annan P., "A review of ground penetrating radar application in civil engineering: a 30-year journey from locating and testing to imaging and diagnosis". NDT \& E International, 96, 2018, pp.58-78.

3. Lalagüe A., Lebens M. A., Hoff I., GrØv E., "Detection of rockfall on a tunnel concrete lining with ground-penetrating radar (GPR)". Rock Mechanics and Rock Engineering, 49(7), 2016, pp.2811-2823.

4. Kilic G., Eren L., "Neural network based inspection of voids and karst conduits in hydro-electric power station tunnels using GPR”. Journal of Applied Geophysics, 151, 2018, pp.194-204.

5. Zhou B., Zhao F., Jiang J., Liu W., "Application of ground penetrating radar in detecting defect of tunnel lining". Journal of Railway Engineering Society, 5, 2012, pp.40-44.

6. Li J., Zeng Z. F., Huang L., Liu F. S., "GPR simulation based on complex frequency shifted recursive integration PML boundary of 3D high order FDTD”. Computers \& geosciences, 49, 2012, pp.121-130.
7. Shu Z. L., Liu X. R., Liu B. X., Wang D. L., "GPR threedimensional forward modeling of defects in tunnel lining and engineering verification". China Railway Science, 34(4), 2013, pp.46-53.

8. Liu Z. H., Wu H., Zhou D., Wei H. Y., "Application of spectrum inversion method in GPR signal processing for tunnel lining detection". Chinese Journal of Geotechnical Engineering, 37(4), 2015, pp.711-717.

9. Gao Y. T., Xu J., Wu S. C., Lu J. H., Chen W., “An intelligent identification method to detect tunnel defects based on the multidimensional analysis of GPR reflections". Chinese Journal of Engineering, 40(3), 2018, pp.293-301.

10. Zhang X. W., Gao Y. Z., Fang G. Y., "Application of Hilbert spectrum analysis in ground penetrating radar thin layer recognition". Chinese Journal of Geophysics, 56(8), 2013, pp. 2790-2798.

11. Zan Y. W, Li Z. L., Su G. F., Zhang Y. Y., "An innovative vehicle-mounted GPR technique for fast and efficient monitoring of tunnel lining structural conditions". Case Studies in Nondestructive Testing and Evaluation, 6, 2016, pp.63-69.

12. Baryshnikov V. D., Khmelin A. P., Denisova E. V., "GPR detection of inhomogeneities in concrete lining of underground tunnels". Journal of Mining Science, 50(1), 2014, pp.25-32. 
Sheng Zhang, Yongsuo Li, Guihai Fu, Wenchao He, Da Hu and Xin Cai/

Journal of Engineering Science and Technology Review 11 (6) (2018) 62 - 69

13. Cui Y., Wang L., Xiao J., "Automatic feature recognition for GPR image processing". World Academy of Science, Engineering and Technology, 61, 2010, pp.176-179.

14. Yu Q. M., Zhou H. L., Wang Y. H., Duan R. X., "Quality monitoring of metro grouting behind segment using ground penetrating radar". Construction and Building Materials, 110 , 2016, pp.189-200.

15. Zhang S., Li Y. S., Fan T. C., Jiang D. Y., Zeng J., "Model experiment and frequency spectrum analysis of detection signal for adverse geological bodies in tunnel". Journal of Hunan City University (Natural Science), 26(4), 2017, pp.1-5.

16. Liu D. K., Ju N. P., Huo Y. X., "Analysis of the spectrum difference of ground penetrating radar (GPR) for different media fillings". Modern Tunnelling Technology, 50(5), 2013, pp.23-28.

17. Li C. M., Wang L. S., Xu M. J., Liu Y. S., Zhong K., "Objects recognition of ground penetrating radar in karst regions using wavelet energy spectrum analysis". Chinese Journal of Geophysics, 49(5), 2006, pp.1499-1504.

18. Wang X. N., Liu S. X., "Noise suppressing and direct wave arrivals removal in GPR data based on Shearlet transform". Signal Processing, 132, 2017, pp.227-242.

19. Ling T. H, Zhang S., Li S. R., "Hilbert-Huang transform method for detection signal of tunnel geological prediction using ground penetrating radar". Chinese Journal of Rock Mechanics and Engineering, 31(7), 2012, pp.1422-1428.

20. Yang Y. Q., He S. H., Qi F. L., Liu J. R., Jiang B., "Simulation tests on GPR detection of composite linings of railway tunnels". Chinese Journal of Geotechnical Engineering, 34(6), 2012, pp.1159-1165.
21. Kravitz B., Mooney M., Karlovsek J. D., "Cavity detection in two-component annulus grout behind a pre-cast segmental tunnel liner using Ground Penetrating Radar". Tunnelling and Underground Space Technology, 83, 2019, pp.381-392.

22. Lai W. W. L., Chang R. K. W., Sham J. F. C., "A blind test of nondestructive underground cavity detection by ground penetrating radar (GPR)". Journal of Applied Geophysics, 149, 2018, pp.10-17.

23. Nobes D. C., "Ground penetrating radar response from cavityspp.A demonstration using a simple model”. NDT \& E International, 91, 2017, pp.47-53.

24. Feng D. S., Dai Q. W., Weng J. B., "Application of multiresolution time domain method in three dimensions forward simulation of ground penetrating radar". Journal of Central South University (Science and Technology), 38(5), 2007, pp.975-980.

25. Feng D., Wang X., Zhang B., "Specific evaluation of tunnel lining multi-defects by all-refined GPR simulation method using hybrid algorithm of FETD and FDTD". Construction and Building Materials, 185, 2018, pp.220-229.

26. Li Y., Li S. C., Xu L., Liu B., Lin C. J., "Forward simulation of ground penetrating radar and its application to detection of tunnel lining diseases". Rock and Soil Mechanics, 37(12), 2016, pp.3627-3634.

27. Zhong S., Wang C. Y., Wu L. X., Tang X. J., Wang Q. Y., "Borehole radar response characteristics of point unfavorable geo-bodies: forward simulation of its surrounding rock and filling condition". Rock and Soil Mechanics, 33(4), 2012, pp.1191-1195.

28. Ahmadi R., Fathianpour N., Norouzi G. H., "Detecting physical and geometrical parameters of some common geotechnical targets through their effects on GPR responses". Arabian Journal of Geosciences, 8(7), 2015, pp.4843-4854. 